\title{
A New Approach to Microsurgical Medical Education: Mobile Phone Repairs
}

\author{
José Henrique Fazzi ${ }^{1 *}$, Marina Schor ${ }^{2}$ and Paulo Schor ${ }^{3}$ \\ ${ }^{1}$ Department of Medicine, Federal University of São Paulo, Brazil \\ ${ }^{2}$ Faculty of Land and Food Systems, University of British Columbia, Vancouver, BC, \\ Canada \\ ${ }^{3}$ Department of Ophthalmology, Federal University of São Paulo, Brazil \\ *Corresponding Author: José Henrique Fazzi, Department of Medicine, Federal \\ University of São Paulo, Brazil.
}

Received: August 07, 2020

Published: August 19, 2020

(C) All rights are reserved by José Henrique Fazzi., et al.

\begin{abstract}
Background: Technology has been increasingly used as a teaching tool in medical education, and simulation training is at the forefront of that shift in teaching methods. This project proposes an innovative tool for training students in microsurgery. Through fixing their cell phones under the guidance of a supervisor, students got a chance to improve their technique in microscope use.

Furthermore, the procedure allowed for students to discuss insecurities surrounding the performance of microsurgery.

Materials and Methods: Eight students from the Federal University of São Paulo were involved in the project and were selected through a phone screening process. Those students brought in their own faulty cell phones which were the main materials used. Other materials included pieces that needed replacing in the phones and the tools needed to open, close and handle the apparatus generally.

Results: $87.5 \%$ of the students achieved the expected result with a successful procedure and $25 \%$ of students had technical problems during the procedure. $75 \%$ of students, however, had no major technical problems during the procedure. Two of the eight students who participated in this project had trouble with the cell phones one month after the procedure. The remaining had fully functional devices.

Conclusion: The majority of students successfully completed the cell phone repairs. Overall, there was a high level of acceptance of the project by students as a successful learning tool which increased both their abilities and self-confidence. Students overall left satisfied with the activity and said they would recommend it to others.
\end{abstract}

Keywords: Surgery Simulation; Medical Training; Ophthalmology; Technique; Practice-based Learning

\section{Introduction}

Each opportunity for health care providers to practice a procedure before moving on to performing it on a human patient increases patient safety [1]. We are in an era of great technological advance in medicine which is accompanied by a myriad of innovative surgical equipment in hospitals. In this context, medical education cannot be left behind, as students can derive great benefit from technological tools for learning. As greatly put by Kinio Anna., et al. "We live in a time when an educational revolution is taking place in most surgical and non-surgical medical specialties.

Simulation models have become increasingly popular as part of many training programs, providing a safe and controlled environment where surgical skills can be acquired, perfected and assessed" [2]. 
That is where this project stems from. We propose the idea of teaching medical students how to perform microsurgery in a nonconventional and motivational way which allows students to train while being able to make mistakes and learn to fix those as they move along. The procedure also aims to stimulate dexterity and allow for students to practice working through a professional preexisting process in a systematic way before moving on to human patients.

Simulation training plays a major role in medicine, especially when it comes to mastering surgical skills as well as gaining confidence in applying the theory learned in a safe environment. In addition, it can help enhance psychomotor skills without endangering patients or students. These learning tools have also been shown to efficiently shorten the learning curve which ultimately leads to a reduction in errors during the procedure performance, thus reducing long-term health costs [3]. While there are multiple benefits to the use of simulation as a training tool, its widespread applications are limited by several factors. First, the price of these machines can be prohibitive. They also require a significant amount of space which can be a problem depending on the environment in which training is taking place. That, not to mention that the training interrupts clinical duties [4].

All these limiting factors are avoided by this project. We are proposing an affordable simulation training option which only requires a broken cell phone in need of repair.

The goal of the project is to support and train medical students in microsurgery through an innovative approach. We aim to enhance dexterity and encourage students to use microscopes as they learn about microsurgery through fixing faulty cell phones.

The process will teach students to practice steps of a professional procedure that needs to be followed in a systematic way; this will teach discipline and encourage students to become more detail-oriented. Furthermore, this exercise will allow for students to work through the anxiety of performing the procedure before moving on to human patients.

\section{Materials and Methods}

\section{Materials}

The materials used in this project as well as their respective uses are listed below.
Curved metal handle: Allows for holding flat wires, removing connections, and picking up any material inside the cell phone (e.g. small screws). Straight metal tweezers are not appropriate for use as they present a risk of damage to the circuit board and flat cable connections due to the lack of curvature.

Anti-static strap: Prevents static which accumulates in body from getting discharged to the conductive electronic components of the cell phone, which could be a source of damage. One end of the strap is placed on the student's wrist and the other is connected to a metal piece such as a table or bench.

Spatula: Used to remove the device's screen. This is done by placing the spatula between the screen and the edges of the body of the device and performing a "lever-like" motion. Also allows for handling of flat cables and of connections.

Torx $0.8 \mathrm{~mm}$ : Used to remove the screws at the bottom of the device.

Philips 0.6 x 25 mm: Used to remove internal screws from the device.

Touch display puller: Used to pull the device's screen up and, with the aid of the spatula, remove the screen. Uses suction force.

Isopropyl alcohol: This product has a high purity level of 99.9\%. It is used for cleaning the circuits as well as flat cable connections. The alcohol also allows for rust removal, which can be effective for devices which have fallen into water.

Talc-free gloves: Used to prevent the oil in the hands from rubbing off on the connectors on the board, causing defects. Gloves must be talc-free because the talc could also cause damage to the circuits.

Microscope and magnifying glasses: Facilitates small movements during the procedure and allows for students to become accostumed to using the equipment.

\section{Methods}

Eight (8) students from the Federal University of São Paulo were selected on a first-come-first-serve basis through a phone call registration process. In order to register, students called the organizer who then recorded any new participants. All the procedures took place at the Ophthalmology Laboratory of the university. 
The project starts with the identification of what is wrong with the cell phone that is brought in by the student involved in the procedure. After noting the problem, the project organizer purchases any pieces of equipment needed to fix the device. Once all the pieces needed are in the hands of the supervisor, the procedure date is scheduled. On the day of the procedure, the student is first briefed by the organizer about the steps that will need to be taken as well as the materials and different risks associated with the project. This allows for everyone to start on an even ground. The student is responsible for preparing the surgical field as well as for gathering the materials needed for the procedure. Once that is done, they wash their hands to avoid any oil getting into the equipment, put on the talc-free gloves, and the anti-static strap.

The first step of the actual procedure is to remove the bottom screws from the device using the Torx $0.8 \mathrm{~mm}$ with the assistance of the microscope for better visualization. Next, the student must remove the device's screen. This is done by holding the screen with the suction cup and then using that to pull it up as the spatula is used to push up the screen in a "lever-like" motion. This is done without the use of the microscope. The third step is to remove the flat connectors with the use of the curved metal handle. After that, any internal screws, battery or buttons are removed using the Philips screwdriver.

If any pieces are malfunctioning or getting in the way of the new piece of equipment that needs to be inserted, they will also be removed at this time. Once that is done, the new equipment piece can be installed, and the phone can be closed.

Throughout this process, if any fragments or particles fall into the device, the student uses isopropyl to clean out the debris and then waits for a few minutes in order to allow for evaporation of the alcohol. As soon as the procedure is done, the student first removes the anti-static strap and then the gloves. Lastly, the final step is to test the device to see if it is functional.

\section{Results}

The results are based on a series of measures such as whether the procedure was successful and the expected result was obtained, whether there were any technical issues during the procedure, as well as the result of the procedure after one month of its completion.
$87.5 \%$ of the students achieved the expected result with a successful procedure while $12.5 \%$ did not. $25 \%$ of students had technical problems during the procedure such as having difficulty with the removal of the screws or with the closing of the device. 75\% of students, however, had no major technical problems during the procedure. Two (2) of the eight (8) students who participated in this project had trouble with the cell phones one month after the procedure. The remaining six (6) had fully functional devices.

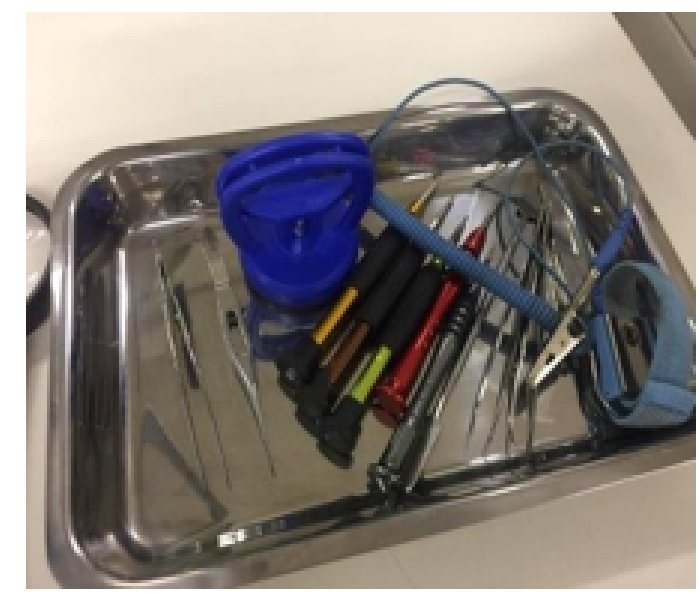

Figure 1: Instrumental tray put together by student.

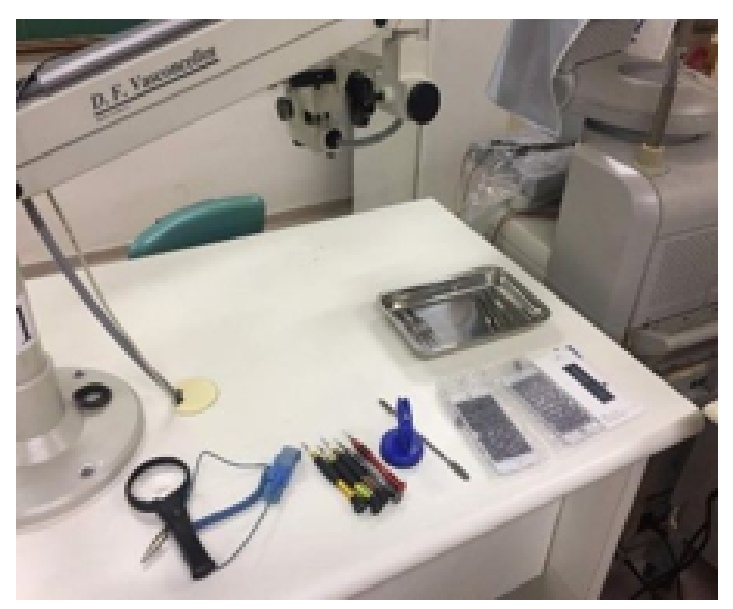

Figure 2: Materials used in this.

Citation: José Henrique Fazzi., et al. "A New Approach to Microsurgical Medical Education: Mobile Phone Repairs". Acta Scientific Ophthalmology 3.9 (2020): 03-08. 


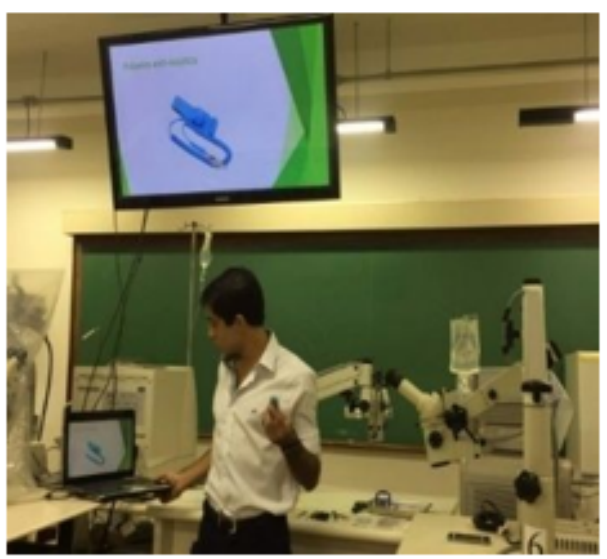

Figure 3: Students being briefed before the procedure.

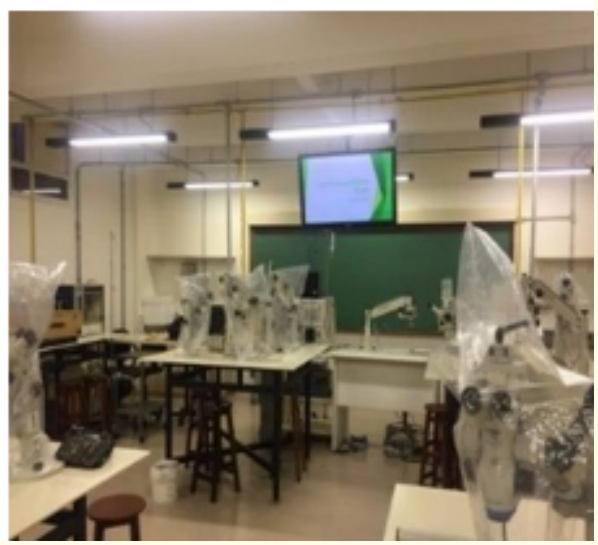

Figure 4: Classroom where the procedure took place.

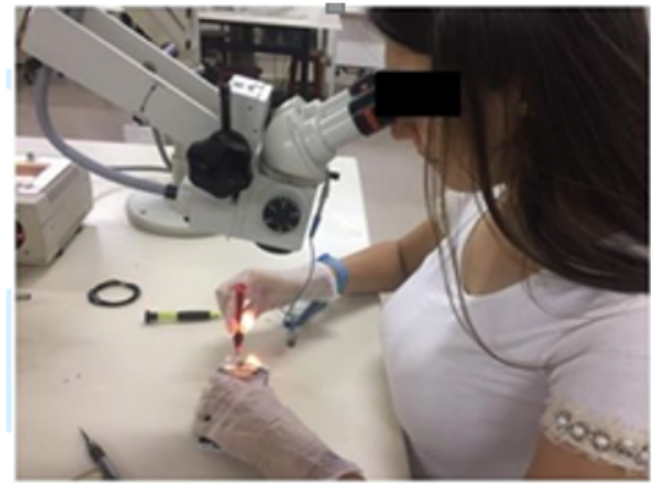

Figure 5: Student during the procedure.

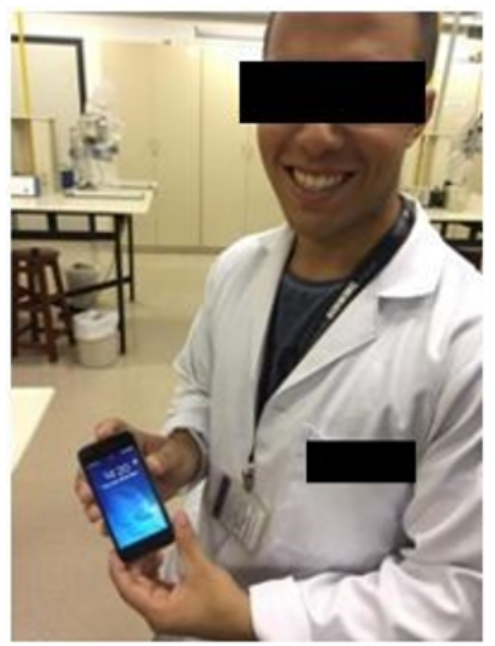

Figure 6: Student after changing the battery of the cell phone.

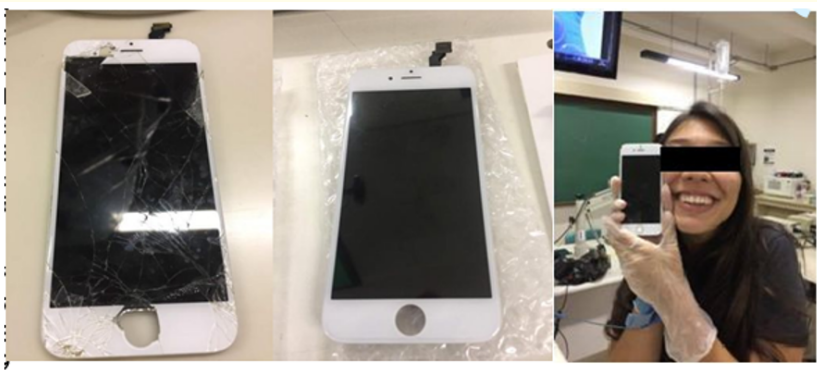

Figure 7: On the left, the old screen. The new screen can be seen in the center and on the right, student after the procedure with cell phone repaired.

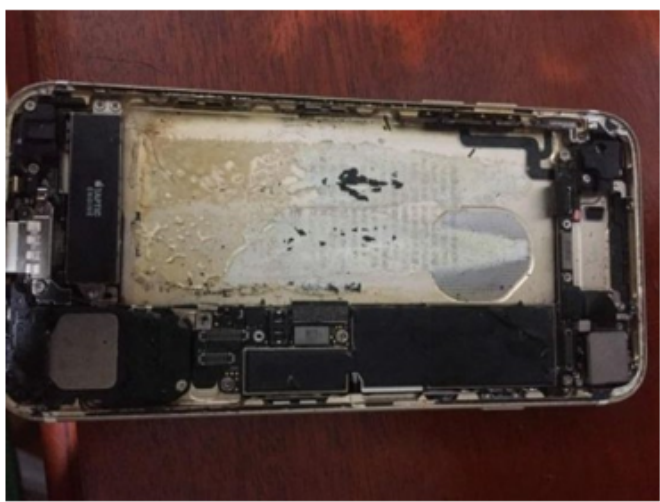

Figure 8: Damaged cell phone with battery removed. 


\section{Discussion}

The students involved in this project worked on their own devices, replacing damaged batteries or screens. After the procedure was completed, the students went on to discuss their experience and psychological aspects of the process such as the fear of possible complications during the procedure and how they thought those emotions would translate to a later real surgery on human patients.

In general, students reported a feeling of euphoria upon completion of a successful procedure. There were also, however, negative emotions that arose during technical difficulties. Some examples of problems that were faced by the students were damage to the wires in the phone structure due to a sudden movement or scraping of the phone structure with sharp instruments. There were also instances where a phone that originally had problems with the screen also had to get its battery replaced unexpectedly. In one particular instance, a student's cell phone started to display more severe problems during the procedure and so a later date was scheduled for the completion of the project in order to allow for new pieces to be acquired.

In the meantime, however, the phone overheated, and the battery melted, rendering it unsalvageable. Options were discussed with the student including whether a new phone would be acquired or if monetary compensation would be distributed by the university's department. The latter was chosen as a resolution, and due to the fact that participants had been instructed to backup their data before the procedures, none of the student's information was lost. All of the unexpected events that students had to handle while fixing their cell phones resembled those which often arise during actual human surgery. Intraoperative problems such as cardiac arrest, ischemia, seizures and hemorrhaging are not uncommon. Performing this procedure on cell phones allowed the students to experience the complications and practice fast-critical thinking without jeopardizing the safety of any human patients.

Another important area of concern for the students related to the performance of the cell phones post-procedure. This also allows for us to draw a parallel to human surgery in terms of the anxiousness of novice surgeons surrounding post-operative complications for patients. As with human surgery, the procedure presented risks for the equipment and some of the cell phones did not respond as expected to the handling. This also exposed students to post-procedure care and maintenance.
It is said that "one quarter of medical students lack awareness of their psychomotor aptitude and many do not feel prepared to participate in the next phase of their training" [5]. With that in mind, this project goes a long way towards assisting students in developing dexterity and gaining confidence while performing microsurgery. A study was conducted by Hanrahan et. al. in which the impact of anxiety-related tremors as well as that of self-perception of anxiety were measured in a simulation scenario. The tremors themselves did not seem to affect the outcome of the procedures being conducted, but the self-perception of anxiety had a significant negative impact on the results of the procedure. This indicates that more confident students perform better in simulated surgical settings as well as in actual surgeries, which strengthens the need for increased exposure to mock procedures [6].

While we acknowledge that there are currently more accurate surgery simulation devices out there such as the "EyeSi" which allows students to practice on the illusion of an eye [7], our project manages to curb the difficulties which come with this simulation platform such as its prohibitive prices. We still stand on the same fundamental idea, that practicing procedures in simulation settings allows for an overall improved performance on actual microsurgeries $[8,9]$. We believe, however, that the fact that students are working on their own phones makes a difference. When using "EyeSi", if a mistake is made, students do not experience any consequences in their lives. When their own devices are on the line, however, the stakes are much higher, and this bring the students closer to experiencing what they would in a regular surgery. Our project also provides the benefit of having a fixed and functional cell phone as the output of the procedure [10].

\section{Student comments}

Below are some comments from students who participated in the project:

- Student 1: "I liked it a lot! The cell phone no longer looks like a boogeyman on the inside, (laughs). And the advisor gave us a lot of support. Really, I was very pleased. I highly recommend the project."

- Student 2: "For me, the project was very useful because it allowed me to fix the cell phone and also made it possible to apply and learn minute techniques to manipulate the different parts under the microscope. All that, not to mention that now the phone is working perfectly. I didn't feel much difficulty at first, but you do have to get used to the parts of the phone and the tools to be able to perform the operation." 
- Student 3 (owner of the unrecoverable cell phone): "My phone was having problems with the touch screen and kept crashing, so when I saw this project, I decided to give it a try. The biggest difficulty for me was having to deal with such small parts and with the fear that I might do something wrong. I do not intend on following the area of microsurgery but found it interesting to participate in the project, even though I could not fix my phone. I would recommend the initiative for simpler cases, as others were successful in their repairs".

\section{Conclusion}

During the project, we were able to help train medical students towards increased dexterity as well as microscope use as they fixed their faulty cell phones. We also provided a platform where the students could follow the steps of a systematic professional procedure and start to work through feelings that might arise and compromise operations done in human patients. Students overall left satisfied with the activity and said they would recommend it to others.

\section{Acknowledgements}

We would like to acknowledge the National Council for Scientific and Technological Development (CNPq).

\section{Disclosure}

The authors disclose no conflicts of interest.

\section{Author Contributions}

- José Henrique Fazzi designed and led the experiments, recruited students and managed the rooms in which the activities took place. Also wrote the article's first draft.

- Marina Schor assisted with data analysis once activities were finalized. Also reviewed, edited and finalized article.

- Paulo Schor helped design the project and acted as supervisor and oversaw every step of the research process.

\section{Bibliography}

1. Hoffman M., et al. "Innovative Simulation Training Models". Disease-a-Month 57.12 (2011): 807-826.

2. Guerrero-González G., et al. "Combined use of simulation and digital technologies for teaching dermatologic surgery". Journal of the European Academy of Dermatology and Venereology 30.12 (2015).
3. Figueiredo E and Oliveira L. "Simulation training methods in neurological surgery". Asian Journal of Neurosurgery 14.2 (2019): 364.

4. Shaikh FM., et al. "Mobile Surgical Skills Education Unit". Simulation in Healthcare". The Journal of the Society for Simulation in Healthcare 6.4 (2011): 226-230.

5. Mitchell PB., et al. "Career Interest and Psychomotor Aptitude Among Medical Students". Journal of Surgical Education 76.6 (2019): 1526-1533.

6. Hanrahan J., et al. "Hands train the brain-what is the role of hand tremor and anxiety in undergraduate microsurgical skills?" Acta Neurochirurgica 160.9 (2018): 1673-1679.

7. Solverson DJ., et al. "Virtual Reality Simulation in Acquiring and Differentiating Basic Ophthalmic Microsurgical Skills". Simulation in Healthcare: The Journal of the Society for Simulation in Healthcare 4.2 (2009): 98-103.

8. Staropoli PC., et al. "Surgical Simulation Training Reduces Intraoperative Cataract Surgery Complications Among Residents". Simulation in Healthcare: The Journal of the Society for Simulation in Healthcare 1 (2017).

9. Feudner EM., et al. "Virtual reality training improves wet-lab performance of capsulorhexis: results of a randomized, controlled study". Graefes Archive for Clinical and Experimental Ophthalmology 247.7 (2009): 955-963.

10. Radia M., et al. "A guide to eyes: ophthalmic simulators". The Bulletin of the Royal College of Surgeons of England 100.4 (2018): 169-171.

\section{Assets from publication with us}

- Prompt Acknowledgement after receiving the article

- Thorough Double blinded peer review

- Rapid Publication

- Issue of Publication Certificate

- High visibility of your Published work

Website: www.actascientific.com/

Submit Article: www.actascientific.com/submission.php

Email us: editor@actascientific.com

Contact us: +919182824667 